Revue d'histoire de l'Amérique française

REYUE D.HISTOIRE DE L'AMÉRIQUE FRANÇAISE

\title{
Un débat parlementaire en 1849
}

\section{Lionel Groulx}

Volume 2, numéro 3, décembre 1948

URI : https://id.erudit.org/iderudit/801476ar

DOI : https://doi.org/10.7202/801476ar

Aller au sommaire du numéro

Éditeur(s)

Institut d'histoire de l'Amérique française

ISSN

0035-2357 (imprimé)

1492-1383 (numérique)

Découvrir la revue

Citer cet article

Groulx, L. (1948). Un débat parlementaire en 1849. Revue d'histoire de

l'Amérique française, 2(3), 375-389. https://doi.org/10.7202/801476ar d'utilisation que vous pouvez consulter en ligne.

https://apropos.erudit.org/fr/usagers/politique-dutilisation/ 


\section{UN DÉBAT PARLEMENTAIRE EN 1849}

D'ici quelque temps on dissertera beaucoup autour des événements pulitiques de 1848 et de 1849 au Canada. Parmi ces faits d'il y a cent ans, un débat parlementaire remua profondément et les galeries de la Chambre et l'opinion publique. Nous voudrions raconter ce débat, en fixer le caractère et les conséquences.

En 1848, la rupture définitive entre Papineau et le ministère Baldwin-LaFontaine, presqu'a demi accomplie, n'appartient plus qu'à ces dénouements dont il ne reste qu'à fixer l'heure. L'ancien chef a précipité la crise avec sa fougue accoutumée. En 1847, s'il parle déjà d'opposition, il parle encore de collaboration. Mais, dès l'année suivante, ses discours et ses écrits éclatent comme une fanfare de guerre: les hommes politiques, quels qu'ils soient, lui sont objets de dégout; la responsabilité ministérielle lui apparaît un insolent " humbug "1. Hommes et régime, Papineau n'épargne ni celui-ci ni ceux-là, en attendant que, l'année suivante, il défende qu'on le confonde avec ceux qu'il appelle résolument les " déserteurs de la cause populaire " ${ }^{2}$.

Du côté du ministère, l'on n'a pas attendu non plus 1848 pour se hérisser d'hostilité. Avec ses allures de pamphlet, le manifeste Papineautiste de 1847 a profondément déplu à Robert Baldwin. Au lieu d'un homme d'État assagi par les événements, il n'avait trouvé, dans ce morceau de littérature électorale, confiait-il à LaFontaine, que " l'ancien et irréductible agitateur $"{ }^{3}$. Les deux hommes allaient se heurter dès l'ouverture de la session de 1848, alors qu'à un caucus du parti libéral, le chef réformiste désavouerait énergiquement un projet d'a-

1. Arch. de l'auteur, Lettre à O'Callaghan, 27 mars 1848. - AC., Corr. ElginGrey, (éditée avec notes et appendice par Sir Arthur Doughty, C.E.B., en 4 tomes), Elgin à Grey, 10 et 18 mai, 6 et 15 juin 1848 .

2. Arch. de l'auteur, Lettre à Madame L.-J. Papineau, 5 nov. 1849.

3. AC., Doc. LaFontaine, Baldwin à LaFontaine, 25 juin 1848. 
mendement de Papineau en réponse au discours de la couronne ${ }^{4}$. D'abord sur la réserve, la presse de langue française n'a pas mieux accueilli ce retour aux vieilles récriminations. Et puisque la violence appelle la violence, dès le mois de mai 1848 , la Revue Canadienne, à la dévotion de LaFontaine, riposte vigoureusement à l'Avenir ${ }^{5}$. A partir de ce moment, le ton de la polémique ne cesse plus de s'envenimer. On se fût cru aux pires jours de 1835, heure des premières divisions dans les rangs du parti national, alors que les frères ennemis se mitraillaient à qui mieux mieux de gros mots et d'injures, comme il arrive toujours, en ces brouilles entre anciens amis ou compagnons d'armes. Comme bien l'on pense, ce ton des journaux a tôt fait de passer aux discours publics et aux manifestes électoraux. En juin 1848, Wolfred Nelson se jette dans une virulente offensive. Il réveille et porte devant le public une vieille querelle qui a déjà traîné, vers 1839 , dans les journaux patriotes et dans les propos des proscrits réfugiés aux États-Unis ${ }^{6}$. Nelson dénonce à ses électeurs ce qu'il appelle la fuite de Papineau en 1837, le matin de la bataille de Saint-Denis. Un déchaînement de furies s'ensuit: échange ininterrompu de démentis, de provocations, d'interpellations sur le mode cambronien; échange aussi d'attestations solennelles, toutes également contradictoires et toutes également assermentées. On ne recule pas devant le duel. Accusé, lui aussi, d'avoir fui à Saint-Charles, Georges-Étienne Cartier échange des balles avec Joseph Doutre ${ }^{7}$. Querelles sans retenue, rageuses, où la vérité historique trouverait moins à gagner que l'échauffement des passions, ${ }^{8}$ et d'où personne ne sortirait grandi. Elgin notait à lord

4. AC., Corr. Elgin-Grey, Elgin à Grey, 2 mars 1848.

5. AC., Corr. Elgin-Grey, Elgin à Grey, 4 mai 1848.

6. Voir Papiers Duvernay dans RAPQ., pour 1926-27 (en particulier p. 214).

7. Fauteux, Aegidius, Le duel au Canada. Collection du Zodiaque " 35 », Montréal 1934, p. 272-78.

8. Sur ce point d'histoire resté obscur et pratiquement insoluble, et qui relève plutôt de la biographie, on pourra consulter le Canadien, 5 juin 1848 :(Manifeste de W. Nelson à ses électeurs); le Canadien, 7 juin 1848: (Riposte de L.-J. Papineau); le Canadien, janvier 1849: plusieurs articles sous ce titre: Quelques reflexions sur la lettre du Réverend M. Chartier. - Voir aussi: Corr. de L.-J. Papineau, lettre à Mme Papineau, 13, 15 oct. 1848; lettre à O'Callaghan, 28 oct. 1848 . Consulter aussi: lettres d'O'Callaghan à Papineau et journaux de l'époque, en particulier, la Minerve, l'Avenir, la Revue Canadienne. Voir surtout, Cahiers des Dix, No 5, article de Montarville Boucher de la Bruère: Louis-Joseph Papineau de Saint-Denis à Paris, p. 79-107. Encore: Résumé impartial de la discussion Papineau-Nelson, sur les événements de 
Grey le secret plaisir des vieux tories au spectacle de cette polémique qui envahissait plus qu'à moitié les journaux de langue française et où deux factions de l'armée rebelle de 1837 et 38 se renvoyaient avec fureur les accusations de poltronnerie ${ }^{9}$. Engagée à l'été de 1848, cette pénible querelle s'est prolongée tout l'automne de 1848. On aperçoit donc en quelle atmosphère enfiévrée va s'ouvrir, à Montréal, le 18 janvier 1849, la session du Parlement.

Mais pour voir plus clair, dans le débat qui s'en vient, peut-être convient-il d'élargir encore ce tableau d'histoire. Comment dire l'ébullition qui agite alors, un peu partout les esprits? 1848, nœud historique, point de rencontre de si graves événements ou évolutions. Ce sont les années où l'Angleterre fait le saut dans le libre-échange économique, au risque d'ébranler son empire colonjal. En Europe, sur le continent, c'est, en France, la révolution de février, victoire de la démocratie sur la monarchie de juillet, secousse qui parut se répercuter d'un monde à l'autre. " L'éclair de nos orages ", devait écrire GarnierPagès, " illumina le monde entier ". Formule d'emphase qui dépassait la vérité, moins encore dans sa forme que dans les faits. A ne s'en tenir qu'au synchronisme historique, Garnier pouvait paraître avoir raison. Les événements de Paris seront suivis, le 13 mars 1848, d'un autre triomphe de la démocratie à Vienne, sur Metternich, le 15 mars, de l'émancipation des Hongrois, le 17 de la révolte de Milan, le 22, de la proclamation de la république à Venise, de la formation d'une assemblée constituante à Berlin le 18 mars, d'un soulèvement des Allemands du Holstein, contre le roi du Danemark (21-24 mars), du Congrès des Slaves à Prague le 2 juin, de l'union des Roumains à Bucarest le 23 juin. Le mouvement - ce que n'ont pas toujours noté les historiens européens - s'étendit au nouveau monde, surtout à l'Amérique latine. " Les constructeurs de l'Amérique latine qui vinrent après Bolivar, s'inspirèrent, dans une large mesure, des idées libérales françaises. Ils

Saint-Denis en 1837, Montréal 1848, 8vo, 16 p. Ce que l'on peut invoquer de plus grave contre le Dr Nelson, c'est qu'il ne juge à propos de donner cette version de la conduite de Papineau à Saint-Denis, que dix ans après les événements et pour des fins de polémique. Un motif patriotique, a-t-il dit, lui a dicté sa première version favorable au grand homme. Soit, mais alors, la seconde version, qui empêche qu'tlle n'ait été dictée au Dr par la partisanerie ou la passion politique?

9. AC., Corr. Elgin-Grey, Elgin à Grey, 24 aout 1848. 
furent particulièrement influencés par les hommes de la révolution de 1848 , dont les croyances politiques se teintaient de la nouvelle philosophie sociale qui donnait préférence aux intérêts de la classe ouvrière... Alphonse de Lamartine fut le maître choisi par la nouvelle génération. De 1848 à 1858 il parut un demi-dieu, " un deuxième Moïse ", d'après une autorité du temps ". ${ }^{10}$ Mais où Garnier-Pagès voyait cause et effet entre la révolution de France et l'ébullition presque universelle, l'histoire est bien obligée de s'en tenir à de simples coïncidences. Tel était le cas, en particulier, des soulèvements européens, antérieurs quelques-uns à 1848, par exemple, à Naples et en Sicile, et beaucoup moins révolutions de caractère politique que national, provoquées par les coryphées de la théorie des races ou de l'idée de nationalité.

$\mathrm{Au}$ Canada que faut-il inscrire au calendrier de 1848 ? L'année mémorable apporta au pays l'autonomie politique: triomphe définitif du " gouvernement responsable ", lien d'importance tranché avec le siège de l'empire. 1848 apporta aussi au Canada le marasme financier, suite et conséquence du libre-échange anglais, et comme prétendu remède à ce marasme, des projets d'annexion aux États-Unis, patrie, proclamait-on, de la stabilité économique et des réussites éblouissantes.

Au Canada français il semble, au premier abord, que le 1848 européen n'art eu de retentjssement que dans l'esprit politique. Ce qu'une jeunesse acclamera par-dessus tout, c'est la révolution de France, et, dans cette révolution, l'essor de la démocratie, expression de la souveraineté populaire. A cette forme de la démocratie, elle se prit à croire comme à une mystique, comme à une foi, seul espoir d'une regénération du monde. ${ }^{11} \mathrm{Et}$, dans cette foi ou cette mystique, un homme dépasse la jeunesse en ferveur naïve, et cet homme qui a passé la soixantaine, n'est nul autre que Louis-Joseph Papineau. Papineau et la jeunesse qui l'entoure se prennent donc à espérer et à préconiser l'orientation du jeune Canada vers les formes de l'État républicain. Ils déclarent désuets « la centralisation et l'arbitraire d'un gouvernement monarchique ". Le suprême espoir de Papineau, c'est de voir " la Patrie consolée et prospère... républicaine un jour ". Et cet avenir, ajoutait-il, " vous le verrez, vous, jeunesse canadienne, si moi-même et

10. Roberts, Adolphe W., Les Français aux Indes occidentales, Les Éditions Variétés, Montréal 1945, p. 333.

11. Groulx, abbé Lionel, Notre Mâ̂tre, le Passé, tome II, p. 213-32: Un mouvement de jeunesse vers 1850 . 
les autres vieux athlètes descendons à la tombe avant l'aurore de ce grand et glorieux jour "). ${ }^{2}$

Dans les aspirations de la jeunesse canadienne-française et dans celles de Papineau, comment ne pas discerner toutefois quelque chose de l'jdéologie qui vient de secouer les nationalités de l'Europe? Le nationalisme est né depuis longtemps, dans le Bas-Canada. Mais ces dernières années l'ont vu singulièrement se fortifier, et moins par des infiltrations européennes que sous la fatale influence du régime politique de 1841. "Le Rapport Durham et l'Union qui suivit ", observe justement un historien anglo-canadien, M. Edgar McInnis, " avajent en fait contribué à revigorer le nationalisme français ".${ }^{13}$ Et ce nationalisme avait repris vigueur, il faut en convenir, par la même méthode que les nationalismes européens: action d'intellectuels, plongées du peuple dans ses traditions et son histoire. M. McInnis souligne, à ce propos, quoique après bien d'autres, l'influence de l'Histoire de Garneau dont le premier volume parut en 1845, et il ajoute: " Au moment où la poignée des écrivains du Canada de langue anglaise cherchaient encore à tâtons une expression nationale qui leur fût propre, le nationalisme canadien-français florissait dans un mouvement littéraire et historique, avec le dessein de fond de maintenir et de renforcer un esprit de séparatisme racial ".${ }^{14}$ Fait notable, en tout cas, que la résolution de la jeunesse démocratique du Bas-Canada de centrer désormais ses aspirations sur le " Rappel de l'Union ", c'est-à-dire, en somme, sur le retour des deux provinces à la séparation de 1791, à l'autonomie politique et nationale du Canada français. Question brûlante que celle-là et bien des fois agitée depuis 1840. Du “ Rappel ", quelques-uns se sont fait un drapeau électoral avant même la mise en opération du régime de l'Union. Aussitôt fondé l'Avenir, les jeunes rédacteurs du journal se serviront du "Rappel " comme d'un cri de guerre. ${ }^{15}$ Et c'est le 15 avril 1848, deux mois après la révolution fran-

12. Voir Ibidem, p. 230.

13. "The Durham Report and the Union that followed had in fact contributed to the consolidation of French nationalism ". - Canada - A Political and Social history, (New York, Toronto 1947), p. 278.

14. « At a time when the handful of writers in English-speaking Canada were still groping toward a distinctive national expression, French Canadian nationalism flowered in a literary and historical movement, the basic purpose of which was to vindicate and strenghten the spirit of racial separatism. " - Ibidem. p. 279.

15. Voir l'Avenir, à partir du 15 avril 1848. 
çaise de février, observons-le en passant, que l'Avenir inaugure tout de bon sa campagne en faveur du " Rappel ". Rentré d'exil et revenu à la politique, Papineau n'a pas médiocrement orienté cette jeunesse vers cette libération politique. Pour lui aussi, le " Rappel " deviendra la réforme radicale, urgente. L'Union, y voit-jl autre chose qu'un régime bon tout au plus à corrompre les hommes politiques en même temps qu'un prolongement détestable du colonialisme? Il écrira bientôt à son fils Amédée (15 juin 1849): "Hors le rappel de l'Union, il n'y a pas de chance à trouver la moindre intégrité politique "). Et quelques mois plus tard (5 nov. 1849), à Mme Papineau: " Je ne puis être jamais réputé Unioniste ni serf volontaire et content du vasselage colonial " ${ }^{16}$ Rien donc de surprenant si, dans le débat qui va s'ouvrir, Papineau jette de nouveau cette question du " Rappel " et pour en faire l'idée centrale et la partie la plus véhémente de ses discours. Discours, du reste, tout pleins d'allusions aux révolutions d'Europe et qui prouvent. combien s'inspirait de ces événements d'outre-mer, la pensée du maître.

Voilà bien, replacé en son atmosphère et dans sa perspective historique, ce tournoi oratoire de 1849 au parlement de Montréal. En réalité c'est tout le régime de 1841 et c'est tout l'avenir politique du Canada qui sont mis en question. Et force est donc de reléguer au second plan les animosités réciproques des orateurs, même s'il importe d'en tenir compte. Harcelé, en effet, par trop de ses amis d'hier qui se livrent au jeu de la meute, Papineau, avons-nous dit, n'a cessé d'incliner vers le rôle d'oppositionniste. Sans doute, les hommes du cabinet auront-ils la dignité et le bon esprit de se tenir en dehors de la querelle Nelson-Papineau. Mais l'ancien chef leur pardonnera mal de mollement refréner la guérilla de leurs francs-tireurs. Dans une de ses répliques à Wolfred Nelson, il écrit à propos des ministres: "Avant l'élection j'avais confiance en eux; aujourd'hui, je n'en ai plus; alors, je louais des masques, aujourd'hui, je blâme des visages ".${ }^{17} \mathrm{Au}$ reste,

16. Archives de l'auteur.

17. L'Avenir, 10 janvier 1849. 
et cela aussi, nous l'avons déjà écrit, Papineau ne manquait pas tout à fait de sujets de plainte à l'égard des jeunes et nouveaux chefs libéraux. ${ }^{18}$ Tous avaient été plus ou moins ses protégés et ses disciples politiques; tous étaient quelque peu redevables de leur précoce renommée au prestige du grand bomme, à l'éclat dont, après vingt ans de travaux, il avait auréolé la cause nationale. Que, dans le secret de son esprit, Papineau entretînt contre LaFontaine des griefs particuliers, il n'est pas téméraire de le penser. Ce dernier gardait, aux yeux du maître, la figure toujours peu sympathique du disciple insubordonné. Après avoir été l'un des plus dociles et des plus enthousiastes parmi les jeunes suivants du tribun, LaFontaine avait été aussi l'un des premiers à se déprendre du sortilège. Aux sessions de 1836 et de 1837, n'a-t-on pas vu le jeune député de Terrebonne braver l'autocrate président de la Chambre du Bas-Canada, esquisser des gestes d'indépendance, entraîner même dans la défection quelques-uns de ses jeunes collègues? Entre les deux hommes, le désaccord s'affirme, dès ce temps-là, non seulement à propos de tactique, mais jusque dans les idées. Aussi intransigeant sur le fond, le disciple ne répugnerait pas à des accommodements sur la forme. Mais surtout il reproche au maître son programme trop abstrait, sans prises suffisantes sur la foule. ${ }^{19}$ Dès ce temps-là, aussi, peut-on dire, le disciple s'essayait au rôle de chef. Pendant qu'à la veille même de la bataille de Saint-Denis, Papineau projetait et commençait d'organiser, sur les rives du Richelieu, une réunion parlementaire plus ou moins clandestine, ${ }^{20}$ l'on se figure avec quel vif déplaisir il avait vu LaFontaine solliciter de lord Gosford une convocation régulière des Chambres. Cette attitude pleine de réserves, chez l'ancien lieutenant, Papineau ne l'avait-il pas retrouvée à sa rentrée au pays? Pendant que d'autres s'empressaient autour du grand homme, souhaitaient son retour à la vie politique, allaient jusqu'à mettre leur comté à sa disposition, LaFontaine, plus méfiant ou plus clairvoyant, se tenait prudemment à distance, évitait de se commettre, et entraînait dans cette méfiance ou suspicion, entre quelques autres, des hommes comme A.-N. Morin. Papineau eut nettement l'impres-

18. Groulx, abbé Lionel, Notre Man̂tre, le Passé, III, p. 245-53. 1837.

19. AC., dans Documents Chapman, Lettre de LaFontaine à Chapman, 17 février

20. Archives de l'auteur, Papineau à O'Callaghan, 28 oct. 1848. 
sion d'être mis en quarantaine et il savait par qui. ${ }^{21}$ Bien des ressentjments poussaient done l'un contre l'autre, le maître et le disciple trop émancipé, mais d'abord, entre ces deux hommes, leurs divergences d'idées qui, depuis 1837, n'avaient cessé de s'accentuer. L'un, et c'était LaFontaine, par la pratique du pouvoir et par ses relations intimes avec Baldwin, avait visiblement évolué vers le type du parlementaire anglais, faisant foi aux institutions britanniques pour la conquête des pleines libertés constitutionnelles. L'autre, et c'était Papineau, les yeux plus que jamais tournés vers Washington et vers Paris, s'orientait de plus en plus vers le type du démocrate à l'américaine ou à la française.

D'autre part, Papineau ne pouvait ignorer à quelle intervention généreuse il devait son retour d'exil. En 1843, sous Metcalfe, LaFontaine s'était noblement entremis en faveur de l'illustre proscrit. Le docteur John Rolph, l'un des chefs de l'insurrection dans le HautCanada, venait d'obtenir son pardon et de rentrer en sa province. LaFontaine ne voulut point que l'ancien chef du Bas-Canada fût traité de façon moins équitable; et il n'avait pas craint de mettre alors en jeu son portefeuille de ministre et sa haute fonction de chef du cabinet. Papineau n'ignorait point cet incident. Mais l'on sait, hélas ! qu'entre les hommes, le souvenir de bienfaits échangés avive aussi bien l'inimitié que l'amitié.

Tout laissait donc prévoir quelque prochaine et éclatante rupture. La rupture se produisit à la fin de janvier 1849, au moment du débat sur l'Adresse, en réponse au Discours de la Couronne. Chambre et public, friands à l'époque de ces sortes de spectacles, assistèrent au duel du tribun et du parlementaire. Papineau inaugurait à proprement parler une nouvelle phase de sa carrière publique. Il ne pouvait faire que son intervention dans le débat ne prît le caractère d'un nouveav manifeste. Il prononça un discours virulent, moins fait pour un parlement que pour la tribune en plein air, harangue longue, décousue. L'orateur s'y prendrait jusqu'à quatre fois pour dire ce qu'il avait à dire; et, pendant douze heures, toutes digues rompues, tantôt en anglais, tantôt en français, le flot coulerait gonflé, infatigable. ${ }^{22}$ La plupart des contemporains ont jugé sévèrement cette éruption d'élo-

21. Arch. de l'auteur. Papineau à O'Callaghan, 19 avril 1848.

22. Voir: La Minerve, 25 et 29 janvier $1849 ; 27$ janvier, 10 février 1849. Gerin-Lajore, Dix ans au Canada de 1940 à 1850. (Québec 1888), p. 491-529. 
quence shez un homme qui avait dépassé la soixantaine. Avec stupeur, l'on constate, en effet, chez Papineau vieillissant, la même impuissance toujours à ramasser sa phrase et ses idées, à refréner une verbosité restée par trop juvénile. Cet homme vient de vivre à Paris sept années de réclusion, de repliement sur soi-même; là-bas, il a pu entendre les plus grands orateurs parlementaires de la monarchie de Juillet; il a fréquenté, dans la capitale française, quelques-uns des plus remarquables esprits de l'époque; rien toutefois de ce séjour ou de ces contacts ne paraît avoir profité à Papineau. Rien qui atteste une plus ferme maîtrise de soi, une plus solide maturité d'esprit. En ce discours de 1849, il déroula la longue procession de ses griefs, quelque chose comme le bataillon en petit des fameuses 92 Résolutions. Il en avait contre l'Union et contre les libéraux convertis au régime; il en avajt contre les chefs du gouvernement dont il flagellait la vénalité; il en avait aussi contre la responsabilité ministérielle et ce qu'il en estimait les funestes conséquences: régime des partis, servilité de la presse ministérielle et des majorités parlementaires, abus du patronage; il trouvait énorme la dépense publique, injustifiable et ruineuse la construction des canaux et des chemins; il en avait enfin contre les gouverneurs qu'il continuait de juger avec ses idées d'bomme de 1830: " Je trouve qu'il y a de la folie pour toute colonie d'être satisfaite de quelque gouverneur que ce soit ") dira-t-il. Et l'on reconnaît en ces formules sa hardiesse coutumière. "Ils sont tous bons, tous mauvais ", continuait l'orateur. "Ce sont des agents de Downing Street qui viennent en passant vous exploiter et qui ne sont bons ou mauvais qu'en autant (sic).que leurs instructions leur donnent ce caractère...; et le plus tôt on cessera de nous en envoyer, le mieux ce sera tant pour la colonie que pour la métropole ". Avec la même superbe, il jugeait les deux partis politiques canadiens sans se priver d'incliner vers ses adversaires de jadis: "Depuis que je suis de retour au pays, j'ai été à portée d'examiner les choses, d'étudier les hommes et je puis dire d'un côté que les torys sont meilleurs que je ne les pensais, et, d'un autre côté, que les libéraux sont loin d'être ce que je les croyais... Ce ministère tory dont je pensais tant de mal, et ce ministère libéral dont j'espérais tant de bien ont tous deux également trompé mes espérances et mes craintes ").

Certes, en cette interminable harangue, tout n'apparait pas également répréhensible. L'orateur sut remercier avec dignité lord 
Elgin de la prononciation du discours de la Couronne en langue française. Il s'éleva contre la nomination de politiciens à la magistrature, contre les lois récentes de l'immigration trop conformes aux désirs du gouvernement impérial. Les parties maîtresses de son discours parurent dirigées toutefois contre les absurdités de l'Union, contre la chimère de soumettre à un même parlement et à une même législation deux provinces aussi disparates que le Haut et le Bas-Canada. Il y revint au moins six fois. Ignorant ou feignant d'ignorer les transformations radicales du régime de 1841, en particulier, le gain du " gouvernement responsable ", et, en cette révolution, le rôle tenu par ses compatriotes, il avait beau jeu à étaler les contradictions des libéraux d'avant 1840 . Il disait, par exemple:

...Cet éloge sans mesure, cet éloge insensé, que nous font nos ministres, de la constitution bâtarde qui a été imposée au pays; cet éloge sans restriction de l'acte d'Union, met les membres du cabinet soi-disant libéral en contradiction directe avec eux-mêmes; il contraste on ne peut plus avec leur passé, avec leurs protestations (quand ces protestations pouvaient leur profiter et les porter au pouvoir) contre l'acte d'Union. Rien de plus opposé, de plus contradictoire, que leur conduite d'alors, et celle d'aujourd'hui; que leurs déclarations sur les hustings et leurs déclarations du jour. Quoi! l'acte d'Union a-t-il done subi des changements tels que nous devions maintenant donner une approbation entière à ce que nous avions été si unanimes d'abord à condamner? Mais on veut faire contraster l'acte d'Union avec l'acte de constitution qui nous régissait auparavant: on met ces deux constitutions en contraste, pourquoi? Pour prouver que celle-ci ne doit plus autoriser, soulever aucune réclamation.

Néanmoins, le 23 juin 1841, les membres libéraux de la Chambre d'alors, qui sont les mêmes que ceux d'aujourd'hui, qui votaient dans un sens en 41 , et qui votent dans un sens contraire en 49 , voulant toujours néanmoins conserver le même nom, ces mêmes membres libéraux s'exprimaient dans les termes suivants:

Nous regrettons que la province du Bas-Canada n'ait pas été consultée sur la constitution qui est substituée à celle qui existait par l'acte de 1791, et qu'il y ait des passages, dans l'acte qui constitue actuellement le gouvernement des Canadas, qui sont contraires à la justice et aux droits communs de sujets britanniques ..$^{23}$

L'Union lui paraît un régime intrinsèquement inique, tout à fait contre-nature:

23. Gerin-I ajoie, A., Dix Ans au Canada, op. cit., p. 493-94. 
...Qu'y a-t-il done de si beau dans cet acte d'Union, pour qu'on s'y attache si fortement? Qu'y a-t-on découvert de si excellent, qu'on n'y avait pas aperçu avant d'être monté au pouvoir?

Pour moi, je n'y vois que des infamies, je n'y vois toujours que des iniquités, toujours qu'une loi de proseription et de tyrannie contre mes compatriotes. Je trouve de l'injustice et de l'oppression dans chacune de ses dispositions... ${ }^{24}$

Et encore:

Je ne m'étendrai pas sur l'acte d'Union, c'est un contre bon sens qui nuit également au Haut et au Bas-Canada, qui met les membres de l'une et de l'autre province dans la plus étrange position. C'est ainsi que les membres pour le Haut-Canada ne peuvent pas comprendre ce qui nous intéresse, et qu'ils sont obligés de législater sans connaissance de cause pour nous, comme nous le sommes pour eux. Avec un pareil système, tous les jours il y aura des fautes grossières de commises, et nous serons sans cesse dans la nécessité de défaire un jour ce que nous aurons fait la veille, tant il est difficile, impossible même, de bien législater pour deux peuples différents de races, de mœurs, de caractère et disséminés sur une si grande étendue territoriale ${ }^{25}$.

En conclusion Papineau opinera pour un Rappel immédiat de l'absurde régime, conclusion qui venait, du reste, à l'appui d'un amendement à l'Adresse de la Chambre:

...Je crois donc que, plus tôt viendra la séparation des deux provinces, mieux ce sera pour les deux partis. Le rappel de l'Union est surtout désirable pour le Bas-Canada. Cette Union nous met, visà-vis du Haut-Canada, dans la même position qu'est l'Irlande visà-vis de l'Angleterre. C'est la partie de toute l'Europe où il meurt actuellement de faim, le plus grand nombre d'hommes. C'est la domination d'un pays sur un autre qui produit de pareils résultats. Tant que nous serons soumis, comme nous le sommes, à la domination du Haut-Canada, nous ne pourrons rien espérer de mieux. On doit done s'empresser de faire cesser un pareil état de choses. Jusqu'à présent l'attention publique s'est abandonnée surtout à la réforme électorale: cela, non pas parce que c'est la seule réforme désirable, mais parce qu'elle est un moyen de conduire au rappel de l'Union. Tous les hommes de bon sens sentent que cette mesure est désirable, sentent la difficulté qu'il y a de législater pour deux pays si différents en tous points. Cette union a été formée dans le but avoué de nuire

24. Ibidem, p. 500.

25. Ibidem, p. 516. 
au peuple canadien. De quel prétexte s'est-on servi pour nous l'imposer? C'est qu'elle devait nécessairement amener l'extension du commerce. On doit voir aujourd'hui qu'on s'est trompé, si c'est là l'idée qu'on avait; que le pays n'a jamais été plus souffrant que depuis l'Union. ${ }^{26}$

On le voit: s'il y a de la paille en ce discours, la paille est quelque fois dorée. Le malheur est qu'il y ait trop peu de grain et que le grain ne soit pas toujours de la meilleure qualité. De ce grand effort d'éloquence, un observateur du dehors, Lord Elgin, trop prévenu ou mal renseigné, écrivait qu'il avait produit peu d'effet, sauf peut-être de la fatigue dans l'auditoire. ${ }^{27}$ Elgin aurait pu retenir que Papineau réussit à se faire écouter longuement et avec respect. " Il avait dans l'attitude, dans le geste, dans la voix", écrit un spectateur, Gérin-Lajoie, "quelque chose de fier, de solennel, qui commandait l'attention "). ${ }^{28}$

La riposte qui suivit diminua de beaucoup l'effet de ce discours, riposte d'un parlementaire d'une logique froide, impitoyable. Après la prolixité redondante, une argumentation concise, serrée qui ramassait la pensée confuse de l'adversaire et, d'un trait incisif, crevait et dissipait les nuées. Peu d'orateurs s'opposaient autant que Papineau et LaFontaine, et devenaient par cela même plus redoutables l'un à l'autre. Tout-puissant sur les foules, le premier perdait ses avantages dans l'enceinte parlementaire. Où les gestes de Papineau paraissaient démesurés, sa voix d'un timbre un peu fort, LaFontaine, d'une parole sobre, précise, contenue, s'ajustait à l'auditoire d'un parlement. Chez l'un comme chez l'autre, l'éloquence était à l'image de l'homme. Papineau avait la taille, la figure et la pose souveraine du tribun; LaFontaine, ainsi nous le décrit un courriériste parlementaire de l'époque, ${ }^{29}$ était de taille moins élancée que solide, de larges épaules,

26. Ibidem, p. 529.

27. AC., Corr. Elgin-Grey, Elgin à Grey, 29 janvier 1849.

28. Gerin-Lajole, A., Dix ans au Canada, op. cit., p. 529.

29. Le Morning Courier, cité par la Minerve, 30 nov. 1843, p. 2, col. 5. 
le cou ramassé, avait la tête " remarquablement bien conformée ", le regard pénétrant, l'œil noir et vif, quoiqu'un peu petit. Doué d'une voix harmonieuse, il s'exprimait avec facilité, sauf en anglais; s'il débute ordinairement en cette langue, il passe peu à peu à son parler maternel et retrouve alors toute son assurance. Que si l'on cherche la caractéristique de cette éloquence, il semble que ce soit l'accent d'autorité. "Lorsqu'il parle ", nous dit le même courriériste, " il le fait avec l'air d'un homme qui remplit un devoir... qui sait qu'il sera écouté et que son avis sera suivi ". LaFontaine ne gesticule point. On dirait un parlementaire anglais. "Il débite ses phrases avec une calme rapidité ", nous dit-on encore, " du ton d'un gouverneur qui lirait le discours du trône "). Mais si LaFontaine n'a reçu en partage, ni le charme ni la fougue oratoire de son rival, en revanche, nous assure un autre contemporain, " aucuns discours ne contenaient plus d'idées justes, plus de bon sens, plus de saine logique que les siens $1 .{ }^{30}$

Dans son procès de l'Union des Canadas, Papineau s'était gardé de noter l'abrogation, en ces dernières années, des articles les plus suspects de la constitution de 1841. LaFontaine, on le pense bien, releva impitoyablement cette ignorance ou cet oubli; et ainsi, et d'un seul coup, s'écroulait, pour une large part, l'argumentation du tribun. Avec la même vigueur LaFontaine dénonça le " système d'opposition à outrance " de son rival; aux conséquences fatales d'un tel système, il opposait les effets de sa politique de ralliement, effets heureux, soutenait-il, pour les Canadiens français et pour Papineau personnellement. Alors, avec une véhémence non dépouillée de cruauté, et où apparaissait quelque survivance du jeune et fougueux pamphlétaire des "Girouettes ", ${ }^{31}$ l'orateur racontait les services que sa qualité d'homme du pouvoir lui avait permis, un jour, de rendre à certain exilé et comment, pour rouvrir à ce dernier les portes du pays, il n'avait pas hésité, lui, homme vénal, à mettre en jeu, sous Metcalfe, son portefeuille de ministre. La sortie s'achevait par une volée de flèches, flèches acérées qui ponctuent d'ordinaire, entre anciens amis, les ruptures définitives:

30. Gérin-Lajoie, A., Dix ans au Canada, p. 268.

31. Les Deux Girouettes ou l'hypocrisic démasquée, (Montréal, mai 1834) 75 p. Cettc brochure - qui est un pamphlet virulent - est dirigée contre Dominique et Charles Mondelet, écuyers, avocats. 
Voilà cependant l'homme qui, obéissant à son ancienne habitude de déverser l'injure et l'outrage, ose, en présence de ces faits, m'accuser, moi, ainsi que mes collègues, de vénalité, d'amour sordide des emplois, de servilité devant le pouvoir ! A l'entendre, lui seul a du dévouement à la patrie ! Je ne lui demande pas de reconnaissance; je n'en demande à personne; mais puisqu'il se dit si vertueux, je lui demande d'être juste, et rien de plus. Est-il capable de l'être? ${ }^{32}$

Ce débat parlementaire ne mériterait pas l'importance que nous venons de lui accorder, s'il n'avait pris, dans l'histoire de l'époque, une large signification. De tous ceux qui entendirent les deux rivaux, personne ne s'y trompa: on avait assisté à beaucoup plus qu'un événement oratoire. Les admirateurs de Papineau, les anciens, se défendirent mal d'un peu de stupeur. La mort dans l'âme, la plupart se voyaient forcés de tourner le dos à leur idole, incapables de reprendre l'histoire, comme leur grand homme, dix ans en arrière. Déception profonde qui s'exprime l'année d'après en ces quelques lignes du chroniqueur du Canadien: " Il est certainement à déplorer que l'homme qui était alors l'homme du peuple, l'intelligence qui fut notre orgueil et notre gloire, soit resté immobile quand tout a remué autour de lui... " 3 3 Parmi les jeunes admirateurs, surtout dans le groupe de l'Avenir, on ne se tint pas de joie et d'admiration. Il manquait un chef à cette jeunesse. Et encore que, pour servir de chef, l'homme eat quelque peu passé l'âge, son nom pouvait se hisser comme un drapeau; ces jeunes le hissèrent à bout de bras, sans faire attention que ce drapeau devenait, pour leurs compatriotes, symbole de division. Jusqu'à cette rupture entre LaFontaine et Papineau, le bloc canadienfrançais, solide comme un roc depuis l'Union, avait à peine connu, sous lord Metcalfe, un léger effritement. Cette fois la fissure y serait et pour y rester et s'élargir. Et la fissure, peu d'historiens, ce nous semble, s'en sont rendu compte, n'allait pas ébranler que le bloc français. En ce débat oratoire de 1849 LaFontaine parut le vainqueur. D'écrasantes majorités ont repoussé les amendements de Papineau. Et

32. Voir texte de ce discours: Gérin-Lajoie, A., Dix ans au Canada, p. 529-37. - L'Avenir, 31 janvier 1849.

33. Le Canadien (Chronique métropolitaine de Toronto), 10 juin 1850. 
pourtant le vrai vainqueur, ce fut le vaincu de ces jours-là. La division parmi ses compatriotes, les luttes fratricides issues de la division, entrèrent, pour une part, dans l'amertume qui devait amener LaFontaine à sa brusque démission de 1851 et à sa sortie de la politique. En divisant les siens, Papineau accrut l'instabilité politique du régime. Toute coalition, toute combinaison de groupes ou de partis devenues éphémères ou impossibles, le gouvernement de la province se vit acculé à l'impasse. Moins de dix ans après 1849 , le régime dut confesser faillite. Une délégation de ministres canadiens se rendaient à Londres à la recherche d'une autre formule de gouvernement. Le grand homme avait donc eu raison contre l'Union et son impossible durée. Mais en 1849 l'on eût bien étonné Papineau si on lui avait dit qu'il ne s'acharnerait à démolir l'Union des Canadas qu'au profit d'un autre plan de lord Durham et conçu pour les mêmes fins que la machine politique de 1840: la fédération des provinces de l'Amérique du Nord britannique.

\section{Lionel Groulx, ptre}

\section{De la Forme LitTtéraire en histoire}

Nous nous garderons de rouvrir à ce propos l'absurde débat: l'histoire est-elle une science ou un art? Qu'on n'aille pas touttefois imaginer non plus que, dans notre pensée, l'historien en possession de son métier se doive reconnaître à son dédain de la composition et du style, ainsi que trop de méchantes langues sont prêtes à l'insinuer. Le talent d'exposition est un don aussi souhaitable chez lui que chez n'importe quel savant; peut-être même lui est-il plus nécessaire encore qu'à beaucoup d'autres. Mais l'objet propre de l'histoire étant la restitution et l'explication du passé, le problème à résoudre est non un problème d'ordre littéraire, mais un problème de méthode, et c'est le seul, bien entendu, qui nous retiendra ici.

(Extrait de: Louis Halphen, Introduction à l' Histoire. Presses Universitaires de France, Paris 1948, p. 36-47). 